This is a postprint version of the following published document:

Cabanelas, J. C., Serrano, B., G. González, M. \& Baselga, J. (2005). Confocal microscopy study of phase morphology evolution in epoxy/ polysiloxane thermosets. Polymer, 46 (17), pp. 6633-6639.

DOI: 10.1016/j.polymer.2005.05.017

(C) Elsevier, 2005

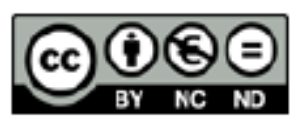

This work is licensed under a Creative Commons Attribution-NonCommercialNoDerivatives 4.0 International License. 


\title{
Confocal microscopy study of phase morphology evolution in epoxy/polysiloxane thermosets
}

\author{
J.C. Cabanelas*, B. Serrano, M.G. Gonzalez, J. Baselga \\ Department of Materials Science and Engineering and Chemical Engineering, Universidad Carlos III de Madrid, 28911 Leganés, Spain
}

\begin{abstract}
The morphology of stoichiometric initially immiscible reactive blends of DGEBA epoxy resin and poly(3aminopropylmethylsiloxane) has been characterized by laser scanning confocal microscopy. Observations were done on samples cured isothermally at different curing temperatures in the range 20-120 8C as well as in situ and in real time at 60

8C. Three different processes were revealed: coalescence, which occurs primarily at very low conversion, diffusion of

DGEBA through polysiloxane-rich domains and chemical reaction, which occurs at the interphase between both phases.

The interphase-thickness and compositional gradients were characterized by laser scanning confocal microscopy

(LSCM). Results show that as curing temperature increases within the studied range, the material becomes more homogeneous although the interphase thickness remains almost constant.
\end{abstract}

Keywords

Reactive compatibilization; Epoxy resin; Confocal microscopy

\section{Introduction}

Addition of third components or modifiers to pseudobinary epoxy systems is a common route to improve the properties of this kind of thermosets. On curing, the initially homogeneous mixture of the epoxy precursors develops a suitable morphology depending on the stoichiometry, thermodynamics and kinetics of the system [1].

There exists a great technical and scientific interest in looking for materials with high toughness and glass transition temperature and with a good adhesion to common reinforcements. Additionally it is desirable to develop epoxy systems with higher reactivity to reduce curing cycles and decrease curing costs and energy, and with an increased hydrophobic character to reduce water absorption (bulk and interphases).

Polysiloxanes seem to be promising as suitable modifiers because of their high thermal and chemical stability, hydrophobicity, transparency, flexibility at low temperatures, high shear strength and high thermal conductivity. Unfortunately polysiloxanes are immiscible with common epoxy resins and show a marked tendency to phase separate forming macrodomains [2]. To overcome this difficulty, functionalised polysiloxanes bearing reactive groups at the chain ends $\left(-\mathrm{OH},-\mathrm{NH}_{2}\right.$, epoxy, ...) have been used [3].

A different approach using perfunctionalized polysiloxanes such as poly(3-aminopropylmethylsiloxane) has been recently reported [4-6]. A complex reactive compatibilization process seems to give rise to quasi-homogeneous fullycured materials but the question about their morphology and its evolution during cure remains open.

The measurement of the silicon content at the surface suggested the existence of macrodomains [6] with a size of about tens of microns in a certain range of curing temperatures and conversions. The big size of this domains and its transparency in the visible range makes the confocal microscopy a suitable technique for morphological studies. It has been used for the evaluation of IPN's structure and for monitoring the spinodal decomposition or surface directed morphology in polymer blends [7,8]. Laser scanning confocal microscopy (LSCM) is also a useful technique for in-depth characterization of the materials and to perform semi-quantitative analysis of the concentration gradients at the phase boundaries.

In this work we will analyse by LSCM the morphology evolution with cure of low molecular weight $\operatorname{poly}(\gamma$ aminopropylmethylsiloxane) mixtures with DGEBA epoxy resin. This analysis will reveal the competition

\footnotetext{
* Corresponding author.

E-mail address: caba@ing.uc3m.es (J.C. Cabanelas).
} 
between chemical reaction and coalescence in fixing the final morphology. The existence of diffuse macrodomains will be experimentally demonstrated and characterized.

\section{Experimental}

\subsection{Materials and samples preparation}

Table 1 shows the chemical formulae and main characteristics of the monomers and polymers used in this study. The diglycidyl ether of bisphenol-A resin (DGEBA) was supplied by GAIRESA (Spain), with a mass per mol of epoxy groups equal to $188.7 \mathrm{~g} \mathrm{~mol}^{-1}$, determined by acid titration. The ratio of secondary hydroxyl groups to epoxy groups was 0.069. The curing agent used was poly(3aminopropylmethylsiloxane) (PAMS), obtained according to a previously published method [4]. PAMS was characterized by vapour pressure osmometry (VPO, Knauer $\mathrm{K} 7000$ ); $M_{\mathrm{n}}$ was $1500 \pm 90 \mathrm{~g} \mathrm{~mol}^{-1}$. Amine hydrogen equivalent was $58.5 \mathrm{~g} \mathrm{~mol}^{-1}$. The oligomer was stored in a desiccator to prevent amine carbonation prior to use.

For confocal microscopy studies, PAMS was labelled with a fluorescent reporter, rhodamine-B sulfonyl chloride (Molecular Probes), dissolving the dye in the oligomer and heating at $60{ }^{\circ} \mathrm{C}$ for $4 \mathrm{~h}$ in $\mathrm{N}_{2}$ atmosphere. Complete sulfonamide formation was checked by size exclusion chromatography. The label concentration in the modified oligomer was $10^{-4} \mathrm{~mol} \mathrm{~kg}^{-1}$.

Curing was performed by mixing previously degassed appropriate amounts of the reactives, stirring at room temperature for $2 \mathrm{~min}$ and curing at desired temperature on the appropriate preheated device. The equivalent ratio epoxy/amine was maintained constant and equal to one in all cases. The curing temperatures were selected between room temperature and $120^{\circ} \mathrm{C}$.

\subsection{Measurements}

Fourier transform infrared spectroscopy in the near range (FTnIR, Perkin-Elmer GX2000, $4 \mathrm{~cm}^{-1}$ resolution) was employed to measure the extent of the epoxy-amine reaction. After mixing, the sample was placed on a preheated sample cell composed of two glass slides and a Teflon spacer $(0.5 \mathrm{~mm})$. Epoxy conversion, $\alpha$, was determined following the extinction of the epoxy band $\left(4532 \mathrm{~cm}^{-1}\right)$ referred to a reference $\mathrm{C} \phi-\mathrm{H}$ band $\left(4623 \mathrm{~cm}^{-1}\right)$, according to the following expression, where $S$ is the integrated absorbance of the band,

$\alpha(t)=1-\frac{S_{4532}(t) / S_{4623}(t)}{S_{4532}(t=0) / S_{4623}(t=0)}$

Thermal transitions on cured samples were determined by differential scanning calorimetry (DSC). A MettlerToledo 822 calorimeter equipped with a liquid nitrogen reservoir was used for low temperature evaluations and a Perkin-Elmer Pyris calorimeter was used for measurements above ambient temperature. Scans were performed in both cases at $10 \mathrm{~K} \mathrm{~min}^{-1}$.

Blends morphology was studied by laser scanning confocal microscopy (LSCM, Zeiss LSCM-5 Pascal). Excitation was carried out with a $25 \mathrm{~mW}$ Ar laser at $488 \mathrm{~nm}$. Emission was detected using a standard dichroic/ filter set. A $20 \times 0.45$ Achroplan objective was used; scanned area was $460 \times 460 \mu \mathrm{m}^{2}$ and resolution of $512 \times$ 512 pixels at 8-bits per pixel (256 channels). All the observations were carried out on samples prepared by putting a drop of resin between two glass slides separated by a $0.14 \mathrm{~mm}$ Teflon spacer, and curing the sample in an oven at the desired temperature. Images were taken after quenching at room temperature. For the 'in situ' study of morphology evolution during curing, the microscope was equipped with a thermostated stage (Linkam Sci. PEI 120).

\section{Results and discussion}

\subsection{Morphology analysis of cured samples}

The final morphology of the thermosets was observed on samples cured isothermally at different temperatures. Curing times were long enough to achieve the limiting six

Table 1

Main characteristics of precursor compounds

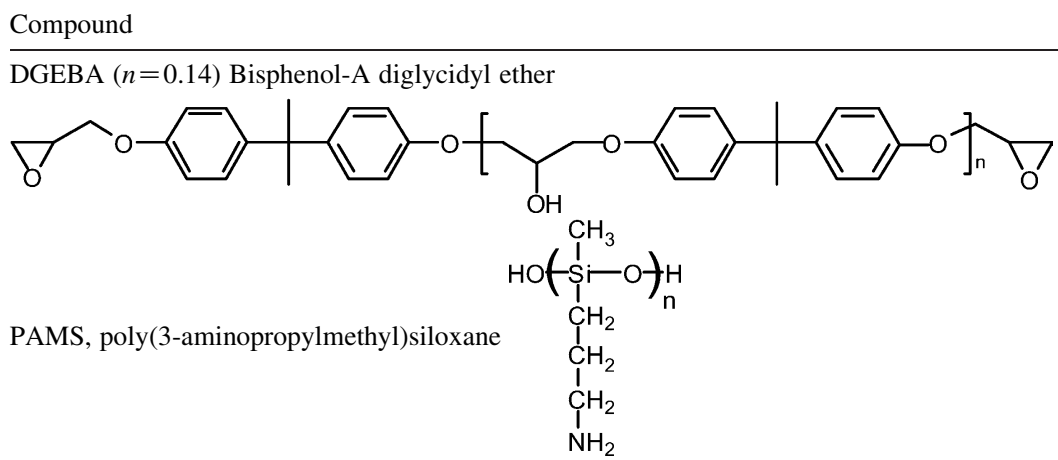


conversion at each temperature. As an example of how conversion proceeds in the curing reaction, Fig. 1 shows the epoxy conversion, measured by FTnIR, as a function of curing time at $60{ }^{\circ} \mathrm{C}$. Similar plots are obtained at higher and lower temperatures in the range $20-120{ }^{\circ} \mathrm{C}$. Although the DGEBA/PAMS systems are initially heterogeneous, no induction period and no evidences of a change in the curing mechanism before vitrification are observed. The kinetic analysis of the curing reaction, reported elsewhere [9], revealed that the first linear portion of the curve can be described by an autocatalysed curing mechanism and diffusion control of the reaction appears well above gelation. Fig. 2 shows the limiting conversion achieved at each curing temperature. It can be clearly observed that systems cured below $90{ }^{\circ} \mathrm{C}$ are vitrified; the high values of the achieved conversion reflect that the reactivity of the system is high even at room temperature. It seems that a limiting conversion of about $0.96 \pm 0.02$ is reached at curing temperatures above $90{ }^{\circ} \mathrm{C}$.

Glass transition temperatures of the samples are presented in Fig. 3. The selected experimental temperature range allows to obtain almost completely cured samples and, therefore, to see the ultimate $T_{\mathrm{g}}$ of the system, located at about $110{ }^{\circ} \mathrm{C}(383 \mathrm{~K})$. At the lowest curing temperatures analysed (below $40^{\circ} \mathrm{C}$ ), two thermal transitions are clearly observed corresponding to the two phases initially present in the mixture: epoxy rich and polysiloxane rich. According to the variation of $T_{\mathrm{g}}$ with curing, that will be presented below, the lower $T_{\mathrm{g}}$ was assigned to the $T_{\mathrm{g}}$ of the epoxy precursor and the higher $T_{\mathrm{g}}$ to the forming thermoset network. When the samples are cured at temperatures above $40{ }^{\circ} \mathrm{C}$, a single $T_{\mathrm{g}}$ is observed suggesting that a more homogeneous material is achieved through reactive compatibilization. This point will be subject of a more detailed discussion further on.

Fig. 4 shows a series of LSCM images of the samples cured at different temperatures. Bright areas correspond to

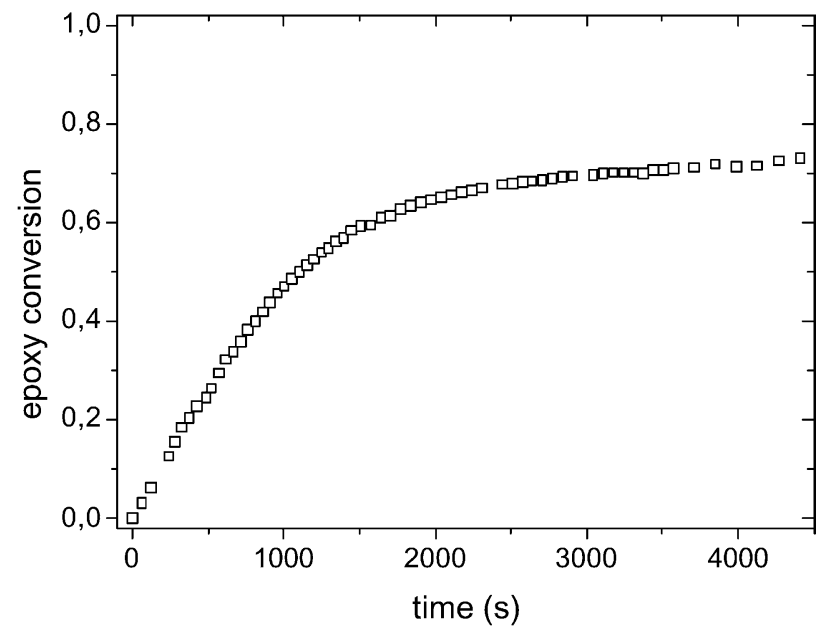

Fig. 1. Epoxy conversion as a function of curing time for an stoichiometric DGEBA/PAMS mixture at $60^{\circ} \mathrm{C}$.

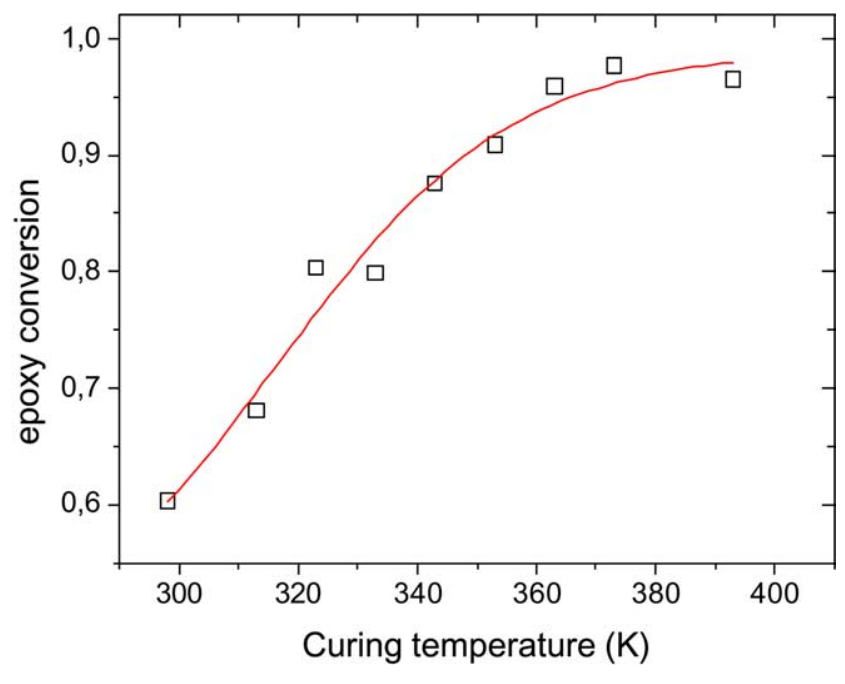

Fig. 2. Epoxy limiting conversion as a function of curing temperature for stoichiometric DGEBA/PAMS mixtures.

regions with high concentration of the rhodamine chromophore, that is to say, to the PAMS-rich regions; dark areas correspond to DGEBA-rich domains. At low curing temperatures (below $40{ }^{\circ} \mathrm{C}$ ), two phases are clearly present: PAMS-rich droplets are dispersed in a continuous epoxyrich matrix. As curing temperature increases, the droplet size of the PAMS-rich domains increases also. Since the mass fraction of PAMS is the same in all cases and all the samples are prepared at the same initial temperature (room temperature), the increase of droplet size takes place by coalescence of smaller particles, a process that is favoured when the viscosity of the matrix is low.

Images taken on samples cured at temperatures above $50{ }^{\circ} \mathrm{C}$ consist of irregularly shaped PAMS-rich domains with diffuse boundaries. The irregular shape suggests that the coalescence process has been slowed down by the competing advance of the reaction, which increases

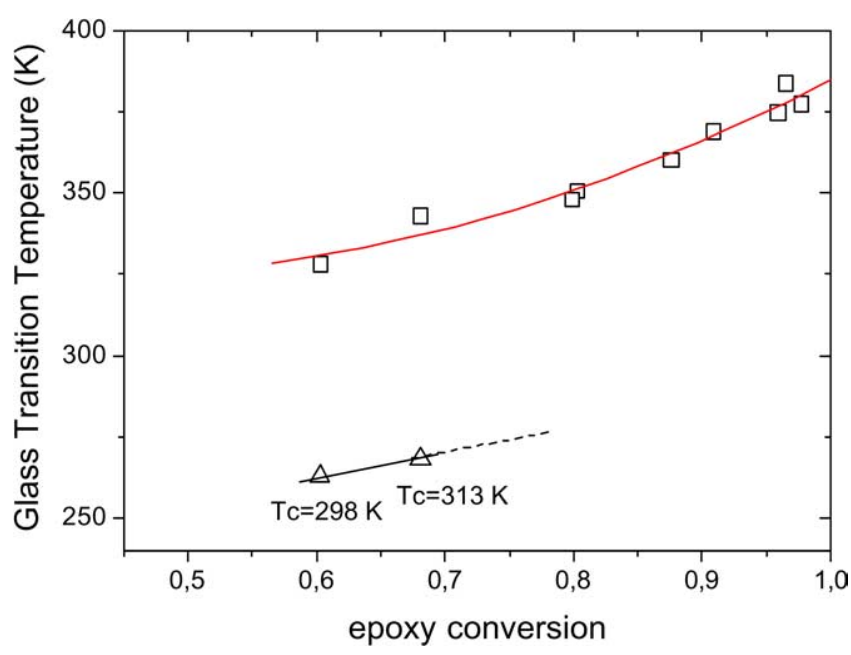

Fig. 3. ( $\square$ ) Highest glass transition temperature obtained at different curing temperatures and presented as a function of epoxy conversion. $(\triangle)$ Low temperature thermal transition (see text for explanation). 
viscosity. The diffuse boundaries are not due to an optical artefact such as lack of focus, and introduce the idea of broad and diffuse interphases between DGEBA-rich and PAMS-rich domains.

The compositional gradient across the boundaries was studied measuring the fluorescence intensity profile along a line that crosses different PAMS-rich domains. Fig. 5 shows the intensity profiles for different curing temperatures. The maximum and minimum intensity values are almost coincident because the scanning conditions were selected in order to maximize the phase contrast in every sample, in such way that values 256 and 1 are assigned to the most intense and less intense pixels of the scanned area. Although this procedure invalidates semi-quantitative analysis based on absolute intensity values, the interphase gradient is better defined.

For low curing temperatures, profiles are sharp and domain sizes are small. As curing temperature increases intensity profiles broaden and larger domains are observed. The interphase-thickness was calculated as the distance between 20 and $80 \%$ of the maximum intensity value. The variation of the interphase-thickness as a function of curing temperature is presented in Fig. 6(a). Mean values were obtained from the average of at least five determinations. It

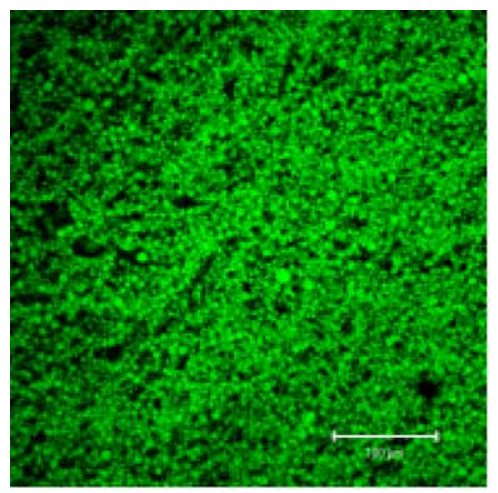

$\mathrm{T}_{\mathrm{C}}=22^{\circ} \mathrm{C}$

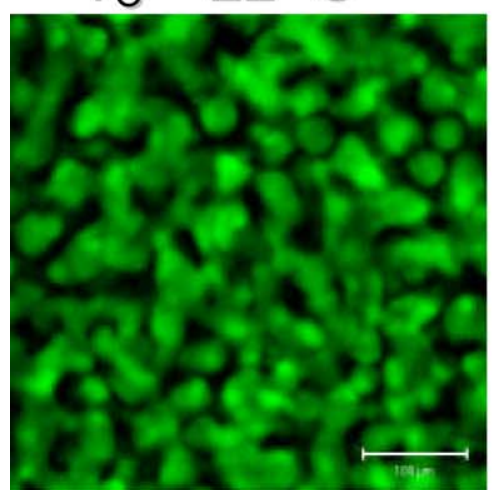

$\mathrm{T}_{\mathrm{C}}=50^{\circ} \mathrm{C}$

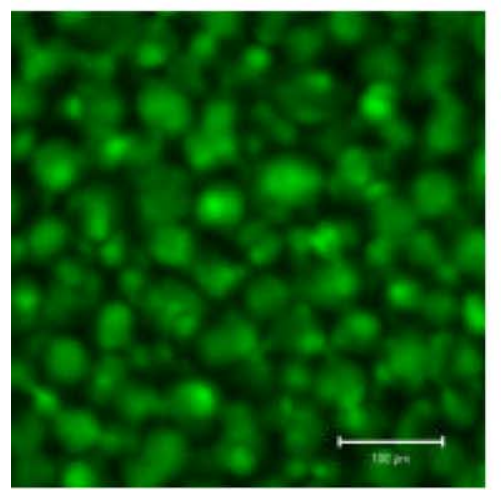

$\mathrm{T}_{\mathrm{C}}=90^{\circ} \mathrm{C}$

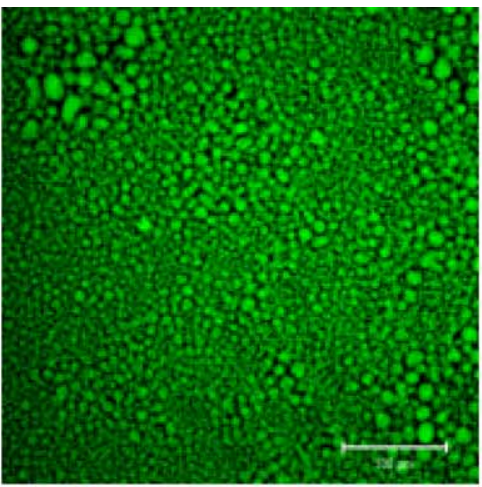

$\mathrm{T}_{\mathrm{C}}=35^{\circ} \mathrm{C}$

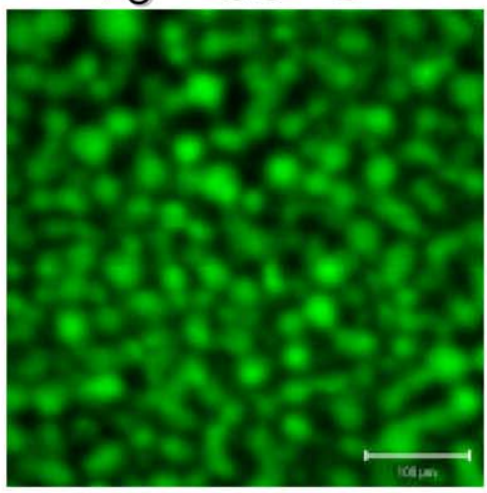

$\mathrm{T}_{\mathrm{C}}=60^{\circ} \mathrm{C}$

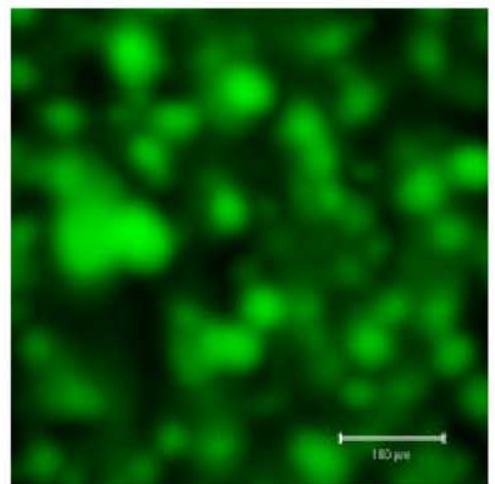

$\mathrm{T}_{\mathrm{C}}=100^{\circ} \mathrm{C}$

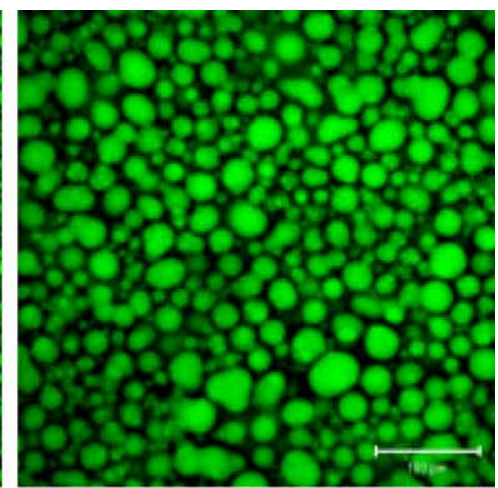

$\mathrm{T}_{\mathrm{C}}=40^{\circ} \mathrm{C}$

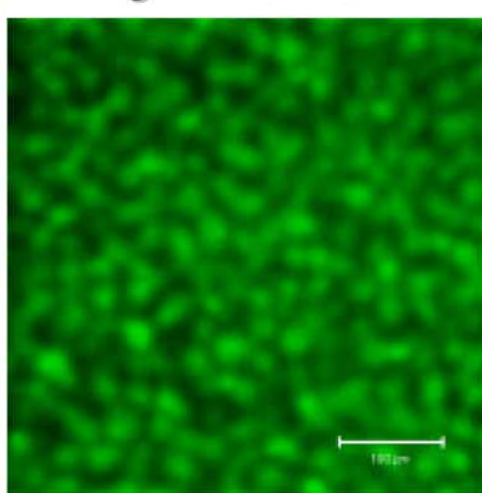

$\mathrm{T}_{\mathrm{C}}=70^{\circ} \mathrm{C}$

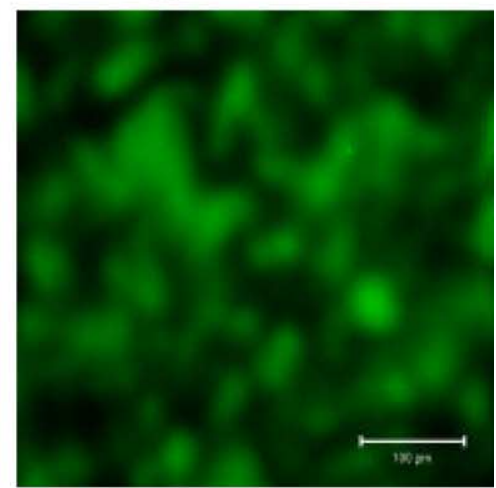

$\mathrm{T}_{\mathrm{C}}=120^{\circ} \mathrm{C}$

Fig. 4. LSCM images of samples cured at different temperatures taken at $50 \mu \mathrm{m}$ from the surface. The scale bar corresponds to $100 \mu \mathrm{m}$. 
can be observed that the interphase width increases from only $2 \mu \mathrm{m}\left(T_{\mathrm{c}}=35^{\circ} \mathrm{C}\right)$ to $15-17 \mu \mathrm{m}\left(T_{\mathrm{c}}=70{ }^{\circ} \mathrm{C}\right)$ and levels off for higher curing temperatures. The latter value is in the order of the half-size of PAMS-rich domains. Additionally to coalescence and chemical reaction, interdiffusion of DGEBA and PAMS is the third process that takes place and explains these results. As curing proceeds the interface grows by interdiffusion of DGEBA and PAMS until vitrification appears stopping both the diffusion and the chemical reaction.

The compositional gradients at the interfaces can be estimated from the slopes of the curves shown in Fig. 5. Results are presented in Fig. 6(b). As curing temperature increases, the compositional differences decrease suggesting that only at very high temperatures homogeneity can be attained.

These morphological results allow a temptative assignment of the thermal relaxations shown in Fig. 3. Since reaction takes place at the interface, the high $T_{\mathrm{g}}$ values should correspond to domains inside the interfacial region. The low $T_{\mathrm{g}}$ values only observed at low curing temperatures should correspond to free DGEBA molecules. At higher curing temperatures the free DGEBA amount should be very small, not enough for being detectable by calorimetric measurements.

\subsection{Morphology development}

Some questions about how this morphology is reached or why the domain size increases with temperature remain open at this point. The morphology variation during the curing process has been monitored in an in situ experiment at an intermediate temperature $\left(60^{\circ} \mathrm{C}\right)$ and the corresponding images are presented in Fig. 7. Coalescence of PAMS rich domains occurs mainly at the beginning of the curing, when viscosity is still low. At epoxy conversions about

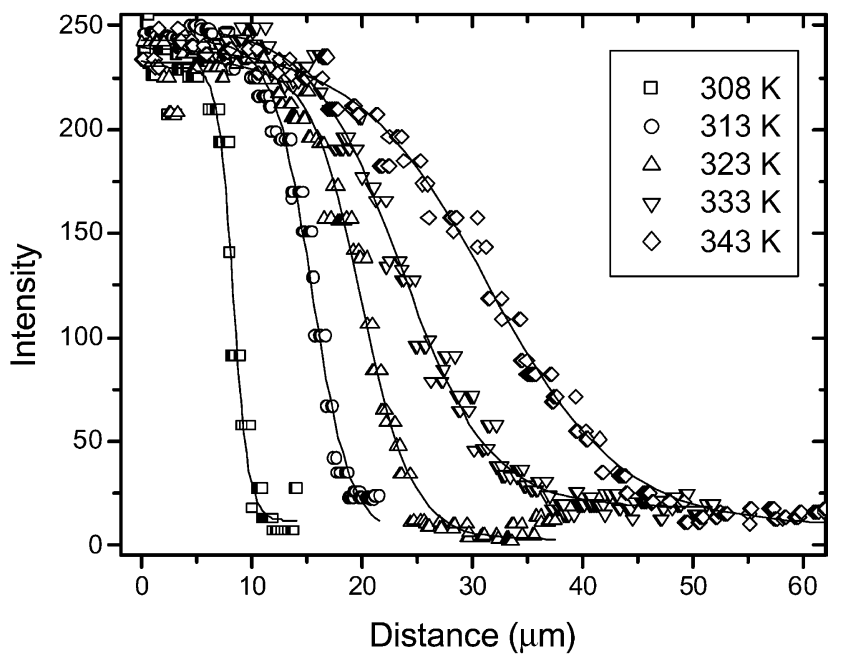

Fig. 5. Intensity profiles along a line crossing a PAMS-rich domain for samples cured at temperatures indicated in the insert. The abscissas origin corresponds to the geometrical centre of a PAMS-rich domain.

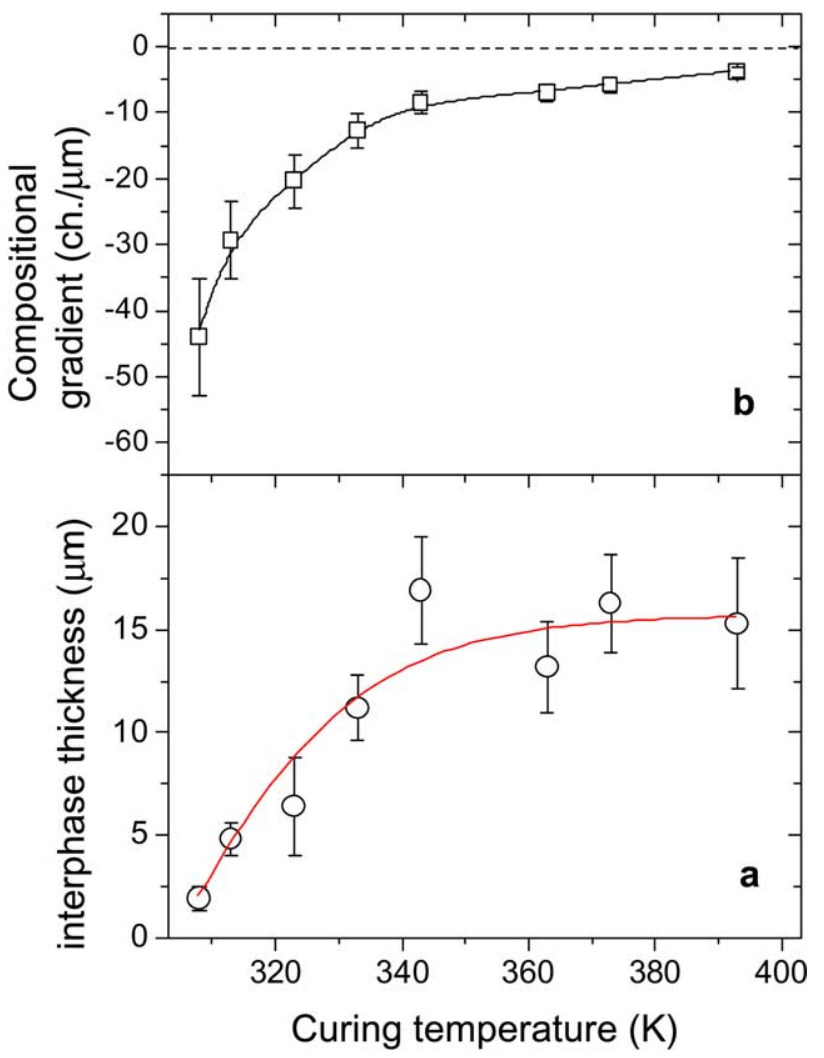

Fig. 6. (a) Mean interphase-thickness (see text) and compositional gradient, (b) as a function of curing temperature.

$20 \%$, the essential morphological features become fixed, and the subsequent reaction advance can only be revealed observing the gradual increase of the interface, which is the result of the interdiffusion of PAMS and DGEBA as mentioned before.

In an heterogeneous system, where reaction takes place at the interphase, stoichiometry is no longer maintained, critical conversion becomes topology dependent and a number of defects can appear [10]. Under stoichiometric conditions, gel conversion should appear around 20\%, according to classical Stockmayer statistics. This value coincides with morphology fixing but gelation cannot explain this fact since unbalanced stoichiometry, expected for an interphase, increases critical conversion and even can suppress it. High viscosity due to extensive branching should be at the origin instead.

At the beginning of the reaction the interfacial energy of PAMS-rich droplets, which consist mainly of pure PAMS, is so high that PAMS domains coalesce reducing the interfacial energy of the system. At the same time, the reaction at the interface reduces the interfacial tension by the grafting of DGEBA molecules into the PAMS chains. The grafted specimen should have a surface tension intermediate between those of the two components, reducing the interfacial tension by a copolymer effect. At a certain conversion, next to gelation, the interfacial tension 

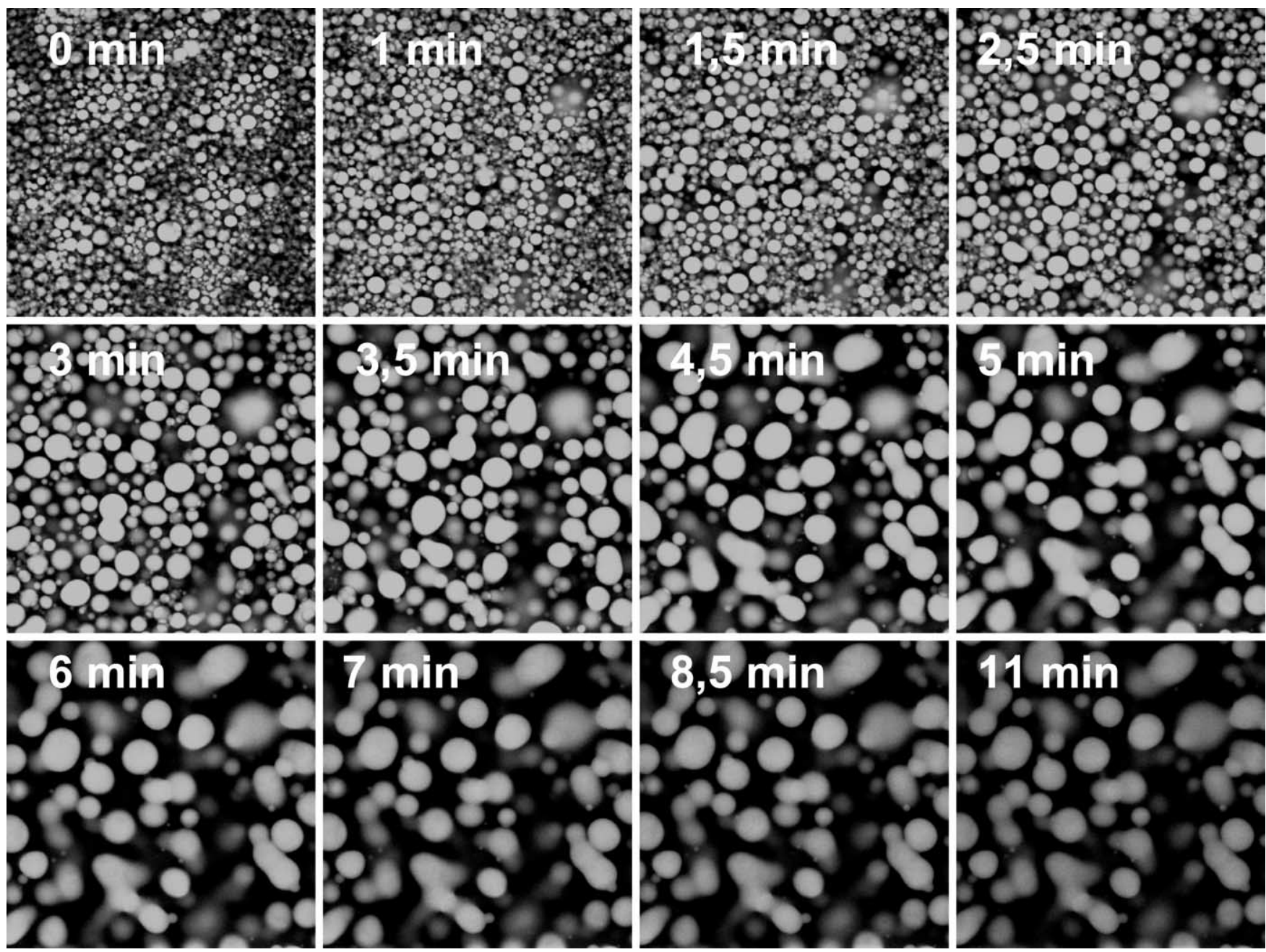

Fig. 7. LSCM in situ monitoring of morphology changes during the cure of an stoichiometric DGEBA/PAMS mixture at $60{ }^{\circ} \mathrm{C}$. Full width of each image corresponds to $460 \mu \mathrm{m}$.

is so low and viscosity so high that the driving force to coalescence becomes reduced fixing the morphology.

To understand more this behaviour, the thermal relaxations of the reactive system were measured at different curing times $\left(\right.$ at $60^{\circ} \mathrm{C}$ ) up to $10 \mathrm{~min}$, which corresponds to about 30\% epoxy conversion (Fig. 1); the results are presented in Fig. 8. Two relaxations are observed in the studied conversion range. At about $255 \mathrm{~K}$ the glass transition temperature of the DGEBA component can be observed, and remains almost constant along the cure process. The relaxation corresponding to the PAMS-rich phase increases very fast even at very low conversions. The reaction progress cannot explain this behaviour and it must be a consequence of fast DGEBA diffusion into the PAMSrich phase. At a given conversion (about $10 \%$ or $5 \mathrm{~min}$ reaction time) the relaxation of the PAMS phase (where the network is forming) reaches the one of the DGEBA phase and even surpasses it. It is exactly at this point when coalescence stops. Unfortunately, this relaxation cannot be measured at higher conversions probably due to the presence of a very broad transition arising from the variable crosslinking degree of the network formed at the interface.

\section{Conclusions}

LSCM is a useful tool to studying the phase morphology in thermosetting heterogeneous blends. The technique allows measuring the interface width, the homogeneity of the material and the morphology changes during the curing process can be analysed in real time.

The cure of an initially immiscible stoichiometric mixture of a polyaminosiloxane and DGEBA is a very complex process that gives rise to a complex and nonhomogeneous morphology. Three different processes have been identified: coalescence, chemical reaction and diffusion. Coalescence occurs spontaneously to reduce the interfacial energy but competes with chemical reaction that reduces the interfacial tension by a copolymer effect. At a certain conversion that should depend on curing 


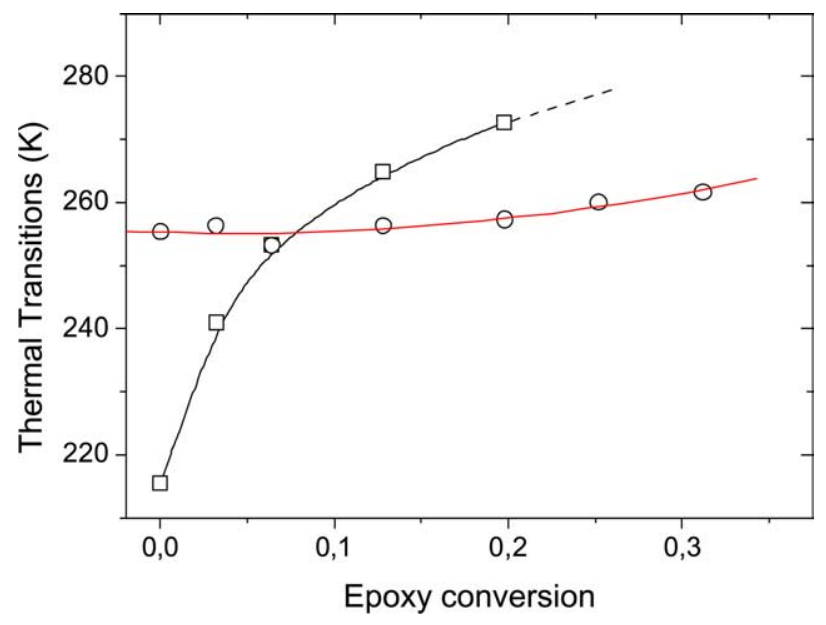

Fig. 8. Thermal transitions for an stoichiometric sample cured isothermally at $60{ }^{\circ} \mathrm{C}$ as a function of epoxy conversion. ( $\left.\bigcirc\right)$ DGEBA phase; $(\square)$ PAMS-rich phase (See text for explanation).

temperature, coalescence process stops and chemical reaction, which does not seem to be controlled by diffusion of reactives, continues to proceed at the PAMS/DGEBA interface. The extended interfacial region has been characterized showing compositional gradients even at the highest curing temperatures, above the ultimate $T_{\mathrm{g}}$ for this system. Once the system vitrifies the width of the interface becomes fixed.

\section{Acknowledgements}

The authors would like to acknowledge the financial support from projects Epoxsil (MAT2000-0391-P4-02) and Fibrodont (MAT2001-0677-P3).

\section{References}

[1] Pascault JP, Sautereau H, Verdu J, Williams RJJ. Thermosetting polymers. New York: Marcel Dekker; 2002. p. 226-247.

[2] Riu CK, Gillham JK, editors. Rubber-modified thermosets resins, vol. 208. Washington: ACS; 1984.

[3] (a) Rey L, Poisson N, Maazouz A, Sautereau H. J Mater Sci 1999;34: 1775-81.

(b) Hou S, Chung Y, Chang C, Kuo P. Polymer 2000;41:3263.

(c) Lee SS, Kim SC. J Appl Polym Sci 1998;69:1291.

[4] Cabanelas JC, Serrano B, González-Benito J, Bravo J, Baselga J. Macromol Rapid Commun 2001;22(9):694-9.

[5] Cabanelas JC, Serrano B, Baselga J. Macromolecule 2005;38(3): 961-70.

[6] Prolongo SG, Cabanelas JC, Baselga J. Macromol Symp 2003;198: 283-93.

[7] Turner JS, Cheng Y-L. Macromolecules 2003;36:1962-6.

[8] Orihara H, Shibuya T, Ujiie S. Polymer 2003;44:8133-7.

[9] González M, Kindelán M, Cabanelas JC, Baselga J. Macromol Symp 2003;200:111-20.

[10] González M, Kadlec P, Stepanek P, Strachota A, Matejka L. Polymer 2004;45:5533. 